\title{
The Fault Diagnosis Research of Gearbox Based on Hilbert-Huang Transform
}

\author{
Cao Fengcai ${ }^{a}$, Pan Hongxia ${ }^{\mathrm{b}}$
}

School of Information and Communication Engineering, North University of China, Taiyuan, Shanxi, 03005I

School of Information and Communication Engineering, North University of China, Taiyuan, Shanxi, 030051

\begin{abstract}
The signal processing based on Hilbert-Huang transform is very suitable for nonlinear and non stationary process; it can extract gear fault features effectively. In this paper we aim at the engineering need of gearbox real-time monitoring and fault diagnosis, expanding a study of JZQ250 Gear Box. We use Hilbert-Huang transform to measure gear vibration signal processing, and use the obtained instantaneous frequency Hilbert marginal spectrum as the fault feature of the gearbox fault diagnosis. Tests showed that, the marginal spectrum based on Hilbert-Huang transform can get the characteristics of fault signal frequency of the gearbox, thus it can identify the type of gearbox fault effectively and achieve early fault prediction. The characteristics of instantaneous frequency can describe the corresponding fault type better. It has a good prospect in the field of gearbox fault diagnosis.
\end{abstract}

Index Terms: Gearbox; Condition monitoring; HHT; Energy-proportion spectrum; Fault diagnosis

(C) 2012 Published by MECS Publisher. Selection and/or peer review under responsibility of the International Conference on E-Business System and Education Technology

\section{Introduction}

The United States Chinese scientists NE Huang proposed Hilbert-Huang transform in 1998, which is a new signal analysis theory. When there is Partial failure on the gear box, the characteristic frequency of vibration and shock are different according to the differences of the defect parts, the characteristic frequency of each component can be informed by the theoretical calculation [1]. The collected gearbox fault signal EMD noise reduction and feature extraction to get IMF group and the Hilbert transform, and the local Hilbert energy spectrum, Hilbert energy spectrum can accurately reflect the role of the frequency with the variation of its energy [2-4]. By spectral analysis can identify fault characteristic frequency spectrum of the energy spectrum which has a large impact, the comparison of the analysis and theoretical calculations can help determine the location and type of the gearbox failure, and to achieve the effective diagnosis of variety typical faults of gear box including the composite failurea[5-6]

\footnotetext{
The project is supported by The Natural Science Foundation of China(Grant No. 50875247)

* Corresponding author.

E-mail address: ${ }^{\text {ccaofengcai@ @uc.edu.cn; }}$ bcaofengcai@yahoo.com.cn
} 


\section{The basic principle of hilbert-huang transformation}

\subsection{Hilbert transformation}

Assigns a continual time signal $x(t)$, it can make Hilbert transform $y(t)$ to:

$$
y(t)=\frac{1}{\pi} \int_{-\infty}^{\infty} \frac{x(\tau)}{t-\tau} d \tau
$$

The $P$ is West tan oak principal value. The transformation can make a conjugate pair to all $x(t)$, $y(t)$ and $z(t)$ :

$$
z(t)=x(t)+i y(t)=a(t) e^{i \theta(t)}
$$

The definition of instantaneous frequency is:

$$
\omega=\frac{d \theta(t)}{d t}
$$

\subsection{The intrinsic mode function}

IMF is a class of signal which meets the physical interpretation of a single component signals, and an intrinsic mode function represents a simple vibration mode compared to the simple monotonic function. An intrinsic mode function should meet the following two conditions: (1) in the whole data segment, the number of extreme points and the number of zero-crossing must be equal or have a difference of one at most; (2) at any time connected, the average of the envelope formed by and the local maximum point and the envelope formed by the local minimum point is zero. That is, the two envelopes on the timeline of local symmetry. [7-8]

Intrinsic mode functions to characterize the volatility inherent in the data model, and each cycle only contains one volatile mode, the phenomenon of ore than one wave mode mixing will not happened; a intrinsic mode function is not binding as a narrow band signal, both frequency modulation and amplitude modulation can work, but also non-steady state; the signal only the frequency or amplitude modulated also can be intrinsic mode function.

\subsection{EMD method}

The instantaneous frequency obtained by Hilbert transform of the intrinsic mode function is meaningful. However, almost every signal data to analyze is not intrinsic mode function, at any point in time, the signal data may include multiple wave modes, so long report that a simple Hilbert transform signal data into can not fully characterize the general frequency. In order to break down the general signal data intrinsic mode function, 
Norden E. Huang proposed EMD method [9-10]. EMD decomposition method used local maximum and minimum values for the envelope. Once all the extreme points, obtained all the local maxima formate the envelope on the data by the cubic spline interpolation function interpolating. At the same time, the next envelope formed by all the local minimum value of the data by interpolation, the envelope and the following are recorded as the average of the envelope $m_{1}$, the original data $\theta(t)$ minus $m_{1}$ get $h_{1}$ :

$$
h=x(t)-m_{1}
$$

Takes $h_{1}$ the original signal, redundant above introduced the step, obtains:

$$
h_{1.1}=h_{1}-m_{1.1}
$$

Screens repeatedly $k$ times, until $h_{1 . k}$ becomes IMF, namely:

$$
h_{1 . k}=h_{1 . k-1}-m_{1 . k}
$$

Written $c_{1}=h_{1 . k}$, then $c_{1}$ is signal $x(t)$, it is the first component that meets the conditions of intrinsic mode function.

For the remainder $r_{1}$ still contains longer period components, it can continue to decompensate $r_{1}$ using the screening process described above in order to obtain the 2-order the intrinsic mode function components, the repetition of this process can obtain a certain number of intrinsic mode functions Component:

$$
\left\{\begin{array}{l}
r_{2}=r_{1}-c_{2} \\
\cdots \cdots \\
r_{n}=r_{n-1}-c_{n}
\end{array}\right.
$$

Signal that obtained ultimately represent intrinsic mode function components and margin of expression of $r_{n}$ :

$$
x(t)=\sum_{i=1}^{n} c_{i}+r_{n}
$$

\subsection{Hilbert spectrum and marginal spectrum}

Do Hilbert transform on all the decomposition of IMF, and ignore the residual component $\mathrm{rn}$, the amplitude distribution at the time - frequency plane is called a Hilbert amplitude spectrum, or simply Hilbert spectrum. Written as: 


$$
H(\omega, t)=\operatorname{Re} \sum_{j=1}^{n} a_{j}(t) \exp \left(i \int \omega_{j}(t) d t\right)
$$

$H(\omega, t)$ reflected the relationship among the signal amplitude, time, and the instantaneous frequency. Hilbert marginal spectrum defined as:

$$
h(\omega)=\int_{-\infty}^{\infty} H(\omega, t) d t
$$

$h(\omega)$ reflected the relationship between signal amplitude and instantaneous frequency.

$E(\omega)$ precisely descript the distribution of the energy of the signal with the instantaneous frequency of the role .

\section{Gear fault diagnosis example}

\subsection{Experimental device}

JZQ250 type of experiment as the research object gearbox, gear box drive and test point arrangement diagram shown in Figure 2, experimental system consists of Y132S-4 three-phase induction motor drives, motor output shaft via the coupling and gearbox input shaft is connected and then the gear box drive, transmission of power to the load wheel, and finally embraced by the hold device.

The key component of the experiment is the gear box, gear box drive and test point arrangement diagram are shown in Figure 1, mainly by the gearbox input shaft, intermediate shaft, output shaft, two pairs of helical gears, bearings and three pairs formed cabinet. By coupling the input shaft is connected with the motor shaft, and supported on bearings on both ends; input shaft transfer power to the intermediate shaft through a pair of helical gears. The intermediate shaft is composed of two helical gears and shafts by a pair of rolling bearings; output shaft is connected to the load wheel button.

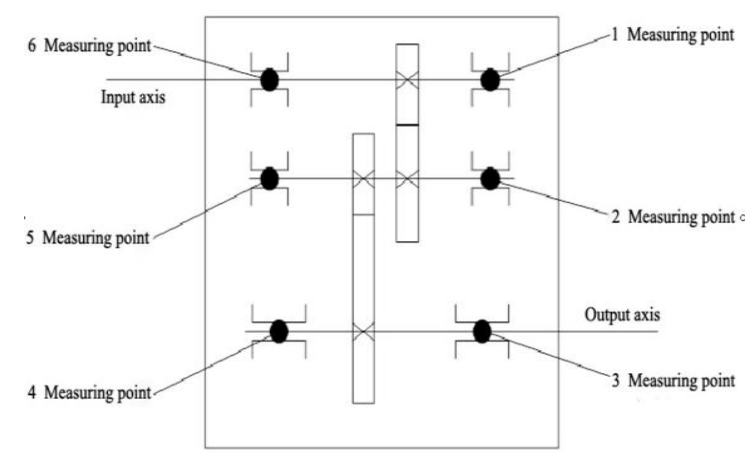

Fig 1. The schematic diagram of gearbox transmission and the test point location 
Table 1 The transfer frequency of each shaft in gear box and meshing frequency

\begin{tabular}{lll}
\hline Ax is & $\begin{array}{l}\text { Rotational } \\
\text { speed }(\mathrm{r} / \mathrm{min})\end{array}$ & $\begin{array}{l}\text { Rotational } \\
\text { frequency } / \mathrm{Hz}\end{array}$ \\
\cline { 3 - 3 } I & 997 & 16.62 \\
II & 316.13 & 5.27 \\
III & 89.26 & 1.49 \\
\hline Gear & Velocity & $\begin{array}{l}\text { Meshing } \\
\text { frequency } / \mathrm{Hz}\end{array}$ \\
\cline { 1 - 2 } $1 / 2$ & ratio z3/z4 & 216.06 \\
$3 / 4$ & $24 / 85$ & 126.48 \\
\hline
\end{tabular}

\subsection{Failure set}

According to the situation of the JZQ250 gearbox in actual use, the intermediate shaft gear box is prone to failure, including the bearing fatigue spelling, cage damage and broken gear tooth phenomenon. The fault data of the experiment is selected when the gear is light load, and simulate gear tooth broken gear exists in 3,4 . And compared with normal conditions, bearing in the gear box above the box to install the six piezoelectric acceleration sensor, 1200rpm motor speed was measured under the six-way vibration acceleration signal. This sampling frequency of the experiment is $20000 \mathrm{~Hz}$, the sampling points are 16384 .

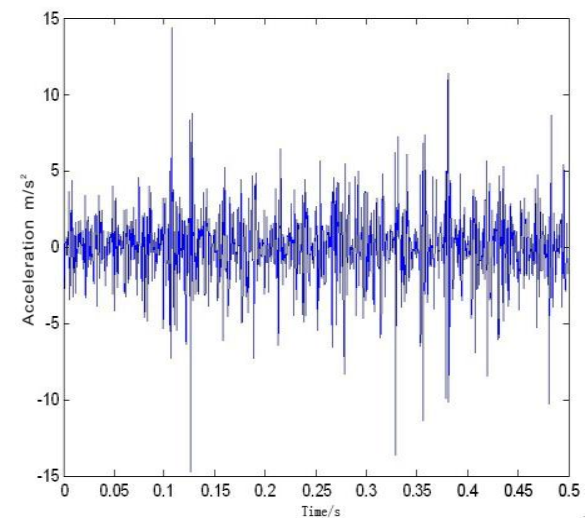

Fig 2. The time-domain waveform of breakage 


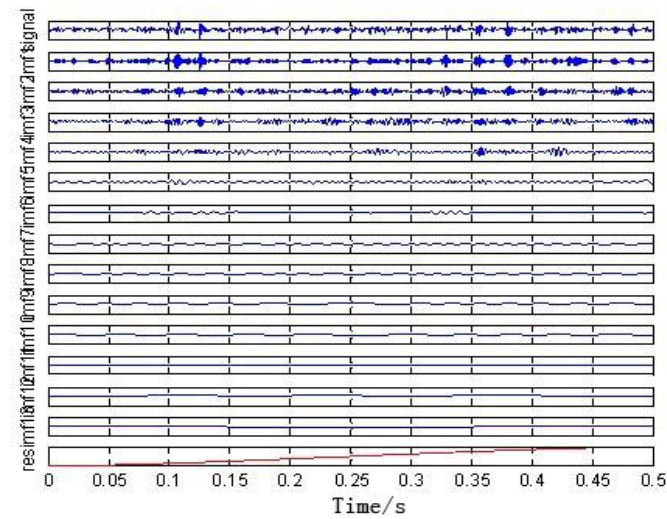

Fig 3. The EMD analysis result of breakage

\subsection{Test data analysis}

As shown in Figure2,it is he time-domain waveform fault when the output shaft gear teeth is broken. Observing the time-domain graph, you can find the waveform of fault signal has approximately periodic pulses, indicating that there is a fault in the area, but can not determine the fault location and type. Shown in Figure 3, it is the decomposition of the fault signal EMD. Observing the resulting IMF is still presenting a certain periodicity, but still can not determine the fault. Hilbert marginal spectrum can be obtained by IMF group on Hilbert transform, shown in Figure4. Marginal in the amplitude spectrum (energy) mainly concentrates within the $500 \mathrm{~Hz}$ band, the band energy analysis, can be (local) energy spectrum, shown in Figure 5. Multiple characteristic lines can be seen from Figure 5, in which the frequency characteristic lines corresponding to the output shaft rotation frequency of $1.8 \mathrm{~Hz}, 7.8 \mathrm{~Hz}, 12.9 \mathrm{~Hz}, 48.1 \mathrm{~Hz}$ octave for the rotation frequency of 4,7 and 25 frequency multiplier, $157.0 \mathrm{~Hz}$ basically consistent with the intermediate shaft and output shaft gear meshing frequency of occurrence of the output shaft can be judged broken teeth fault, the results is consistent with the actual situation.

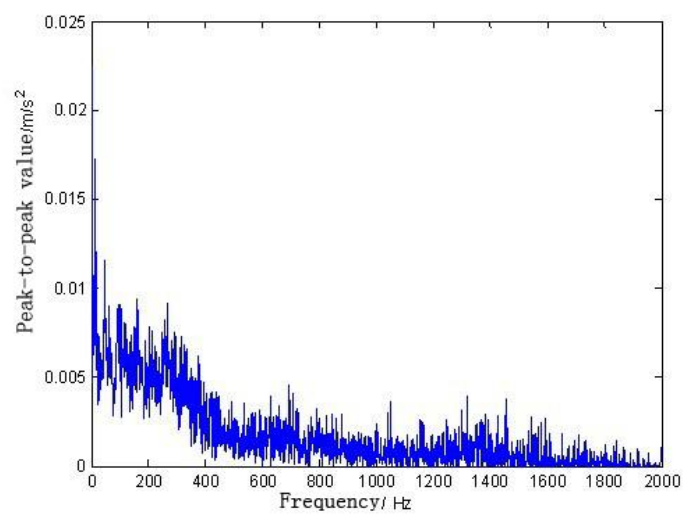

Fig 4. Fig.4 the Marginal spectrum of breakage signal 


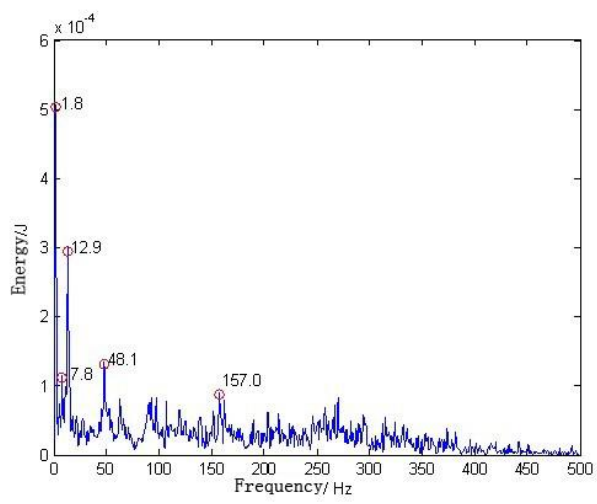

Fig 5. The local energy spectrum of breakage signal

\section{Conclusion}

This thesis introduces the Hilbert-Huang Transform to gear diagnosis and proposes the marginal Spectral Diagnosis based on Hilbert-Huang Transform. The Hilbert-Huang Transform-time adaptive signal processing method is very suitable for nonlinear and non-stationary process, which can be extract gear fault features effectively. The marginal spectrum based on Hilbert-Huang Transform can obtain the characteristics of fault signal frequency of gearbox, which can identify the type of gear box failure effectively.

\section{References}

[1] S R Long, N E Huang. On the Normalized Hilbert Transform and Its Applications in Remote Sensing. Signal And Image Processing for Remote Sensing. New York: CRC Press New York. 2006:3-22.

[2] Yu Dejie, Cheng Junsheng, Yang Yu. Application of EMD method and Hilbert spectrum to the fault diagnosis of roller bearings. Mechanical Systems and Signal Processing, 2005,19(2): 259-270. (In Chinese)

[3] B. Liu, S. Riemenschneider, Y. Xu. Gearbox fault diagnosis using empirical mode decomposition and Hilbert spectrum. Mechanical Systems and Signal Processing. 2006(20):718-734.

[4] Hao Yunhu. The application of wavelet transform in gearbox fault diagnosis. Taiyuan: Master thesis of NUC, 2009

[5] Huang N E,Shen Z, Long S R. The Empirical Mode Composition and the Hilbert Spectrum for Nonlinear and Nonstationary Time Series Analysis [J]. Proc R Soc London, 1998, 454: 903-995.

[6] Shorey A, Kordonski W, Tricard M. The application of Hilbert-Huang transform energy spectrum in bearing fault diagnosis [J]. Journal of Ordnance Engineering College2005, 17(4):37-40

[7] Xiang Ling,Zhu Yongli,Tang Guiji. The application of HHT in the Rotor Fault Diagnosis[J]. Proceedings of the CSEE, 2007, 27(35): 84-88. (In Chinese)

[8] Huang N E, Shen Z, Long S R. A New View of Non- linear Waves: The Hilbert Spectrum[J]. Annual Review Fluid Mechanics, 1999, 31(5): 417-457.

[9] Y.Tani and K. Kawata. The theory and application of the machinery's non-stationary signal diagnosis [M].Beijing: Higher Education Press, 2001

[10] Willian Kordonski. Rub-impact fault diagnosis of the rotor systems based on EMD[J].Mechanism and Machine Theory,2009,44:784-791. 